\title{
Massive barium sulfate aspiration during upper gastrointestinal contrast material study in an elderly patient with dysphagia
}

\author{
Hae Ryong Yun, Chang-gon Kim, Jihye Park, Yong eun Park, Yong-il lee, Byung-Woo Yoo, \\ Kyung Soo Chung, Young Sam Kim, Joo Han Song \\ Division of Pulmonology, Department of Internal Medicine, The Institute of Chest Diseases, \\ Yonsei University College of Medicine, Seoul, Korea
}

\begin{abstract}
Barium sulfate is an inert material used as a radiographic contrast medium during upper gastrointestinal contrast studies for evaluation of patients with dysphagia. Oral barium aspiration is an uncommon but wellreported complication of this procedure. While barium aspiration of small amounts may not cause any symptoms, massive barium aspiration can be life-threatening, particularly in elderly patients with multiple comorbidities. In this case report, we describe an elderly patient with multiple comorbidities who presented with thyrotoxicosis and dysphagia, and then died after massive barium aspiration. Despite administration of intensive medical care with ventilator support and therapeutic bronchoalveolar lavage to remove the aspirated barium, the patient died of multiple organ failure 9 days after barium aspiration. Clinicians should pay attention to elderly patients with predisposing factors for aspiration in whom upper gastrointestinal barium contrast studies are indicated, and should consider other diagnostic tools for evaluation of dysphagia in this population.
\end{abstract}

Keywords: Aspiration, Barium sulfate; Multiple organ failure

\section{INTRODUCTION}

A barium swallowing test is generally safe, but aspiration during upper gastrointestinal contrast material studies is a well-reported complication [1]. However, occurrence of massive barium aspiration can be life-threatening with a mortality rate exceeding 50\% from the development of shock, secondary pneumonia, or acute respiratory failure [2].

We describe an elderly patient with multiple comorbidities

Received: June 19, 2015, Revised: August 07, 2015 Accepted: August 11, 2015

Corresponding Author: Joo Han Song, Division of Pulmonology, Department of Internal Medicine, Severance Hospital, Institute of Chest Diseases, Yonsei University College of Medicine, 50-1 Yonsei-ro, Seodaemun-gu, Seoul 03722, Korea

Tel: +82-2-2228-1930, Fax: +82-2-393-6884

E-mail: augustin76md@yuhs.ac who presented with uncontrolled thyrotoxicosis with dysphagia in whom the aspiration of large amounts of barium during an upper gastrointestinal radiographic contrast study led to a fatal clinical course.

\section{CASE}

A 66-year-old man presented to the emergency department with a 2-week history of progressively worsening difficulty with swallowing foods and liquids, and intermittent nasal regurgitation. The patient reported associated symptoms of generalized weakness, palpitation, and dyspnea, but denied diplopia, dysarthria, and extremity weakness or numbness. He has been diagnosed with Graves' disease for 30 years and had several episodes of thyrotoxicosis with congestive heart failure and atrial fibrillation requiring hospitalization. His other medical history included alcoholic liver cirrhosis, chro-

Copyright ( 12016 Yeungnam University College of Medicine

This is an Open Access article distributed under the terms of the Creative Commons Attribution Non-Commercial License (http://creativecommons.org/licenses/by-nc/4.0/) which permits unrestricted non-commercial use, distribution, and reproduction in any medium, provided the original work is properly cited. 
nic obstructive pulmonary disease, and mitral valve replacement. Three months earlier, he had undergone radioiodine I-131 treatment, and has been managed on a saturated solution of potassium iodide and propranolol.

On physical examination the patient was alert without signs of baseline cognitive impairment. He was afebrile with a pulse of 143 beats per minute, respirations of 20 beats per minute, pulse oximetry of $97 \%$ on room air, and blood pressure $140 / 60 \mathrm{mmHg}$. After an evaluation of his laboratory values, the patient was diagnosed with thyrotoxicosis based on the results of thyroid function test, which showed thyroid stimulating hormone $0.025 \mathrm{mIU} / \mathrm{mL}$ (range, 0.35-4.94 mIU/ $\mathrm{mL}$ ), free T4 $5.86 \mathrm{ng} / \mathrm{dL}$ (range, $0.70-1.48 \mathrm{ng} / \mathrm{dL}$ ), and total T3 $8.0 \mathrm{ng} / \mathrm{mL}$ (range, $0.58-1.59 \mathrm{ng} / \mathrm{mL}$ ), and was admitted for management of thyrotoxicosis and nutritional support.

Despite the addition of methimazole $30 \mathrm{mg} 3$ times daily upon admission, weakness and dysphagia progressed. On hospital day 2, a barium esophagogram was performed for evaluation of dysphagia (Fig. 1). During the study, he aspirated a large amount of barium sulfate, and became distressed with oxygen saturation of $87 \%$ at room air. On examination, his temperature was $36.7^{\circ} \mathrm{C}$; pulse 110 beats per minute; blood pressure $110 / 70 \mathrm{mmHg}$; respiratory rate 30 breaths per minute.
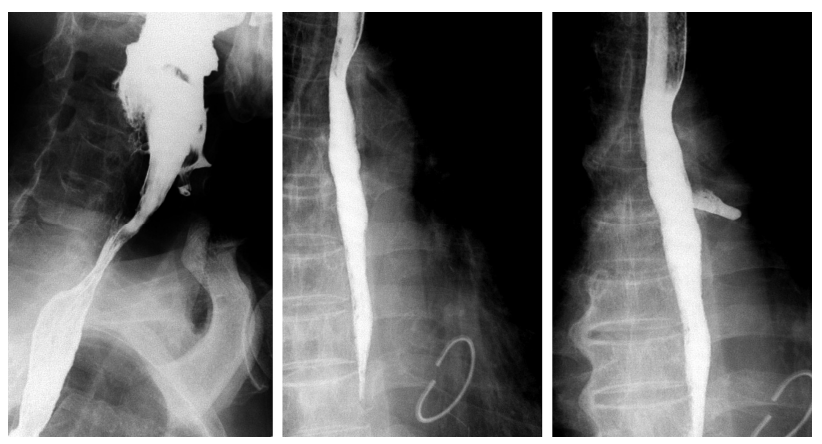

Fig. 1. Esophagography. Single contrast study showed minimal aspiration but no significant filling defect or transition delay.

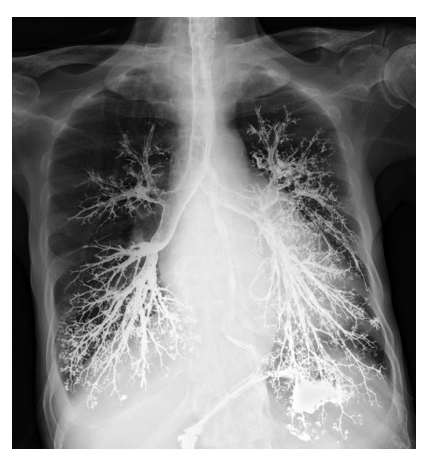

Fig. 2. After barium aspiration, chest X-ray showed endobronchial deposition of barium sulfate in the whole lung.
His oxygen saturation improved to $90 \%$ with a reservoir mask of oxygen flow rate of $15 \mathrm{~L}$ per minute, but showed signs of accessory respiratory muscle retractions. While the esophagogram showed no significant filling defect, the chest radiograph performed after the esophagogram demonstrated a fulminant endobronchial deposition of barium sulfate, highlighting his bilateral airways and bronchial tree of the whole lung (Fig. 2).

Due to the worsening hypoxia, the patient was transferred to the medical intensive care unit (ICU), where he underwent endotracheal intubation and mechanical ventilator support at 30 minutes after barium aspiration. Thirteen hours after intubation he remained in severe respiratory failure with high ventilator requirements (oxygen saturation below 90\%). Therapeutic bronchoscopy was performed and large amounts of barium sulfate were removed during lavage. However, complete removal of aspirated barium contrasts was not feasible because the lavage was limited by hypoxemia. Tracheo-esophageal fistula was not seen on bronchoscopy. Despite the continued intensive care with fluid resuscitation, vasoactive agents, antibiotics, and renal replacement support, the patient died of multiple organ dysfunction in ICU on day 9.

\section{DISCUSSION}

Metabolic myopathy affects $60-80 \%$ of thyrotoxic patients and men are affected more commonly than women [3]. While an enlarging cervical or retrosternal goiter may cause direct impingement of the esophagus, muscle weakness from thyrotoxicosis can cause bulbar muscle wasting, resulting in oropharyngeal or esophageal dysmotility [4,5]. It is usually associated with thyroid crises or chronic thyrotoxic myopathy and acute bulbar palsy, abrupt in onset and fatal, is a rare pattern [5,6]. Oropharyngeal weakness causing dysarthria and dysphagia can be seen with myasthenia gravis, which can develop in $3-10 \%$ of patients with hyperthyroidism, or thyrotoxic hypokalemic periodic paralysis, therefore it is important to distinguish these entities [7].

In the elderly population, the influence of the normal aging process on the oropharyngeal swallow by age-related alterations in somatosensory cortical activation during swallowing [8] and impairment of pharyngeal bolus clearance associated with concomitant medical illnesses [9] has been well described.

On the other hand, there are different comorbid disease 
associations with dysphagia based on age, and their prevalence varies among age groups [6]. In particular, the prevalence of dysphagia associated with esophageal malignancy ranges from $53-93 \%$ with the average age of 63-65 years [10]. Therefore, evaluation to rule out esophageal structural lesions is often the first step in studies to evaluate a cause of dysphagia following detailed history taking and physical examination, particularly in an elderly population.

Among various diagnostic techniques, the initial test is often a barium swallow or an endoscopic examination. In cases of dysphagia to both solids and liquids and suspicion of a motor disorder, as in our patient, a barium swallow provides more useful information by evaluating both esophageal structure and peristalsis [11]. Although rare, oral barium sulfate aspiration is a well-documented complication of upper gastrointestinal studies using contrast material. Barium sulfate, a relatively insoluble salt form of barium, is an inert material that does not usually cause significant chemical pneumonitis [12], thus it is used as a radiographic contrast media which produces "white-out" appearance on chest radiographs. In fact, earlier reports on patients undergoing inhalation bronchography with barium contrast before bronchoscopy was available suggested negligible impact of barium on pulmonary tissue [13,14]. Conversely, Voloudaki et al. suggested that barium particles seem to be phagocytosed by alveolar macrophages, which can potentially cause interstitial fibrosis, seen as centrilobular micronodules on high resolution computed tomography scan [15].

Aspiration of small amounts of barium during diagnostic procedures is occasionally reported, usually from incidental detection on routine chest radiograph without causing any symptoms. Nevertheless, barium aspiration can be varied by the individual status of oropharyngeal functional integrity which can be affected by various conditions, including age, neuromuscular dysfunction, alcoholism, malignancy, and psychological illnesses, as well as the concentration or volume of barium aspirated [16]. A large amount of contrast aspiration can disturb the gas exchange through occupying alveolar spaces, causing a shunt effect and ventilation/perfusion mismatch [17]. In fact, combined aspiration of gastric contents and large amounts of barium can lead to life-threatening conditions with a mortality rate of $30-50 \%[2,18-20]$. According to the limited number of reported cases, barium aspiration seems to be more problematic with greater morbidity and mortality in elderly patients with comorbidities [18].

In the current patient, the main cause of dysphagia appears to be metabolic myopathy involving oropharyngeal and/or esophageal segments from uncontrolled thyrotoxicosis. This and multiple other comorbidities of the patient may have contributed to the massive aspiration that occurred during barium esophagogram. In addition, inefficient expectoration of the aspirate due to his worsening condition may have aggravated the lung lesion, despite the postural drainage maneuver. There is little data on the optimal management for barium aspiration following a contrast study. Empirical use of antibiotics is recommended since aspirate may contain gastric content. In cases of massive barium aspiration affecting almost the entire respiratory trees, therapeutic use of fiberoptic bronchoalveolar lavage to remove the barium may be helpful, although there are opposing views regarding this therapeutic approach concerning the possibility of further dissemination of the barium [16].

Barium swallowing test is a useful diagnostic tool for evaluation of dysphagia. Despite the inert character of barium sulfate per se, massive aspiration of barium is usually accompanied by aspiration of gastric content and can be potentially lifethreatening, particularly in patients with multiple underlying disorders. Although postural techniques may minimize the risk of aspiration during barium studies, other diagnostic tools for evaluation of dysphagia should be considered in elderly patients with multiple comorbidities associated with increased risk of aspiration.

\section{REFERENCES}

1. Pracy JP, Montgomery PQ, Reading N. Acute pneumonitis caused by low density barium sulphate aspiration. J Laryngol Otol 1993;107:347-8.

2. Franquet T, Giménez A, Rosón N, Torrubia S, Sabaté JM, Pérez C. Aspiration diseases: findings, pitfalls, and differential diagnosis. Radiographics 2000;20:673-85.

3. Cook IJ. Oropharyngeal dysphagia. Gastroenterol Clin North Am 2009;38:411-31.

4. Ramsay ID. Muscle dysfunction in hyperthyroidism. Lancet 1966;2(7470):931-4.

5. Chiu WY, Yang CC, Huang IC, Huang TS. Dysphagia as a manifestation of thyrotoxicosis: report of three cases and literature review. Dysphagia 2004;19:120-4.

6. Boddu NJ, Badireddi S, Straub KD, Schwankhaus J, Jagana R. Acute thyrotoxic bulbar myopathy with encephalopathic behaviour: an uncommon complication of hyperthyroidism. Case Rep Endocrinol 2013;2013:369807. 
7. Okada H, Yoshioka K. Thyrotoxicosis complicated with dysphagia. Intern Med 2009;48:1243-5.

8. Teismann IK, Steinstraeter O, Schwindt W, Ringelstein EB, Pantev C, Dziewas R. Age-related changes in cortical swallowing processing. Neurobiol Aging 2010;31:1044-50.

9. Kendall KA, Leonard RJ, McKenzie S. Common medical conditions in the elderly: impact on pharyngeal bolus transit. Dysphagia 2004;19:71-7.

10. Roden DF, Altman KW. Causes of dysphagia among different age groups: a systematic review of the literature. Otolaryngol Clin North Am 2013;46:965-87.

11. Aslam M, Vaezi MF. Dysphagia in the elderly. Gastroenterol Hepatol (N Y) 2013;9:784-95.

12. Willson JK, Rubin PS, McGee TM. The effects of barium sulfate on the lungs: a clinical and experimental study. Am J Roentgenol Radium Ther Nucl Med 1959;82:84-94.

13. Nelson SW, Christoforidis A, Pratt PC. Barium sulfate and bismuth subcarbonate suspensions as bronchographic contrast media. Radiology 1959;72:829-38.

14. Shook CD, Felson B. Inhalation bronchography. Chest 1970;
58:333-7.

15. Voloudaki A, Ergazakis N, Gourtsoyiannis N. Late changes in barium sulfate aspiration: HRCT features. Eur Radiol 2003; 13:2226-9.

16. Jackson M, Kapur N, Goyal V, Choo K, Sarikwal A, Masters IB, et al. Barium aspiration in an infant: a case report and review of management. Front Pediatr 2014;2:37.

17. Chiu CY, Wong KS, Tsai MH. Massive aspiration of barium sulfate during an upper gastrointestinal examination in a child with dysphagia. Int J Pediatr Otorhinolaryngol 2005;69:541-4.

18. Gray C, Sivaloganathan S, Simpkins KC. Aspiration of highdensity barium contrast medium causing acute pulmonary inflammation--report of two fatal cases in elderly women with disordered swallowing. Clin Radiol 1989;40:397-400.

19. Fruchter O, Dragu R. Images in clinical medicine. A deadly examination. N Engl J Med 2003;348:1016.

20. Rodríguez-Gutiérrez R, Mendoza-Garcia A, Gonzalez-Saldivar G, Gonzalez-Gonzalez JG. Massive barium aspiration. Am J Med Sci 2014;348:249. 